\title{
Pedestrian Flow Characteristics at Upstream and Downstream of Bottleneck for Unidirectional Flow under Normal Conditions
}

\author{
Mr. Siddhartha Gulhare', Ms. Aparna P M², Dr. Ashish Verma ${ }^{3}$ \\ ${ }^{1,2,3}$ Department of Civil Engineering \\ Indian Institute of Science \\ Bangalore, India. \\ siddharthagulhare89@gmail.com; aparna.pras@gmail.com; ashishv@iiisc.ac.in
}

\begin{abstract}
The study of pedestrian flow characteristics at upstream and downstream of bottlenecks is important from level of service and evacuation perspective. Many controlled laboratory experiments have been conducted to study pedestrians' behavior at bottlenecks. However, it is unclear whether experiments can reproduce real crowd flow characteristics. In this paper, real field data was collected at normal conditions for unidirectional pedestrian movement at Mahakaleshwar, a Hindu temple at Ujjain, India during Mahashivaratri, a festival day on which a large number of pilgrims visited the temple. Along the corridor there is a width reduction at a U-turn which creates a bottleneck. It is necessary to study pedestrian flow characteristics at bottlenecks to ensure desired level of service at temple premises during heavy flow. The speed-density relationships of upstream and downstream sections were compared and it was found that flow behavior at both the sections of bottleneck severely differ from each other. Pedestrians in the upstream are either at free flow speed for very low density values or moving slowly for intermediate to high range of density values. From the speed-density relationship, it can be concluded that pedestrians at upstream had visual clues of congestion ahead at bottleneck (pedestrian could also see the downstream flow through barricades). Therefore, pedestrians wait at their position, stay in their comfort zone and do not push each other. Thus, even at intermediate local density, pedestrians have such low speeds. This violates the general assumption that pedestrians change their speed only at the shockwave boundary. The movement of pedestrians at upstream is governed by local density and information of congestion status ahead, whereas pedestrian movement at downstream is governed by factors like density, side friction and pedestrians' willingness to compensate for the delay at bottleneck. This study is expected to have application in planning and operation of pedestrian facilities.
\end{abstract}

Keywords: Bottleneck, Pedestrian Flow Characteristics, Speed-Density Relationships

\section{Introduction}

India is a country, which celebrates many religious festivals every year. Being the second most populous country in the world with majority of them having faith in religion/tradition and improved transportation facilities are some of the reasons why India witnesses mass religious gatherings, especially during festival days. This in turns impose huge challenges to organizers to manage such large crowd and to avoid any crowd risk situations.

The current practice of crowd management and crowd control is based on experience or "rule of thumb".. Many event managers including festival organizers in temples, create very long and narrow makeshift corridors to channel pedestrian flow and to avoid random movement of pedestrians in the premises. However, a proper scientific guideline can significantly aid crowd management. With the advancement in computing, it is also feasible to use pedestrian simulation tools to assist in crowd control. The simulation tools can also aid in evaluating various crowd management strategies in advance, prior to the event. 
One significant hindrance to steady pedestrian flow is the presence of bottlenecks. A bottleneck typically refers to a limited area (in general sense) of reduced capacity or increased demand such as onramp [1]. Pedestrian bottlenecks are generally formed due to capacity reduction. It is necessary to study pedestrian flow characteristics at bottlenecks to ensure desired level of service at temple premises during heavy flow. Simulation tools can quantify the impact of bottleneck on pedestrian flow and aid in exploring solutions to reduce its impact. These simulation tools need to be calibrated and validated with empirical data before application. However, there is a lack of pedestrian empirical data around bottleneck in real conditions. This paper attempts to study the pedestrian flow characteristics at the upstream and downstream of bottleneck in a real crowd situation for unidirectional flow.

The outline of this paper is as follows. The next section discusses the review of literature. The section 3 discusses about site condition and data collection. The data analysis is mentioned in section 4 and results are discussed in section 5

\section{Review of Literature}

The study of pedestrian bottleneck is crucial from the perspective of level of service and evacuation. In the last few years, bottleneck and its impact on flow characteristics of pedestrians have gained significant attention. Several controlled experiments in laboratory conditions have been performed at normal conditions to gain more insights [1-3]. Some experimental studies were performed at various stress levels, as an attempt to replicate real field conditions [4]. However, the ability of participants in such experiments to reproduce real behavior and motivation levels of crowd even at normal condition is doubtful. Also in a laboratory setup, one can test combinations of experimental variables, whereas in a field data collection the conditions may not always be favorable.

Pedestrian flow characteristics at bottlenecks are sufficiently researched, but little is known about pedestrian flow characteristics at upstream and downstream of bottlenecks, especially under real conditions. [5] have quantitatively shown that pedestrians' behavior at upstream severely differs from the behavior at downstream and that density and walking velocity are not always linearly related to each other. However, their results were derived from a controlled experiment, where a group of participants were instructed (repeated many times) to move from a waiting area to the other side of the wall through an opening of different widths of bottleneck. In their experiment, while movements upstream of a bottleneck is governed by their information with respect to the traffic state in the bottleneck ahead of them, their movements downstream of the bottleneck seem to be governed by the physical restrictions of the current local situation only. [4] indicated that participants in upstream respond to the visual clues of traffic congestion at bottleneck. [6] studied the flow characteristics at upstream of bottleneck of real pedestrian movement in a tapering walkway. Their results indicate that pedestrians recognized that, a faster speed would hasten their joining at the rear of the queue and maintaining a low speed provided comfortable low density conditions. When congestion was forming, speed in each section of the study area began to decrease before density began to rise and they concluded that the pedestrians respond to the visual downstream congestions. From the review of literature, it was learnt that the impact of bottleneck at upstream and downstream of real continuous pedestrian flow (with varying flow rate with time) has not been quantitatively studied so far. This study attempts to examine flow characteristics at upstream and downstream for real pedestrian flow.

Researchers have reported that pedestrian flow characteristics are influenced by physical and psychological characteristics such as age and gender [7, 8], culture [9], luggage-carrying [10] etc. Some other factors include geometrical and environmental conditions such as roughness or gradient of surface [11], indoor/outdoor walkway [12] etc. The present study compares the speed-density relationship to comprehensively understand the differences in the macroscopic behavior of pedestrian at upstream and downstream. Since, both upstream and downstream sections are studied for the same time period; same set of pedestrians had crossed both sections. Therefore, psychological and physical characteristics of pedestrians is same for both the sections. Similarly, environmental conditions and surface characteristics were also same for both the sections. It was also crosschecked that no other bottlenecks are present ahead 
in the downstream. The pedestrian flow rate at exit of temple was checked and found to be approximately equal to rate of flow at the study area. This also confirms the absence of any other obstruction in the downstream which might have impacted the outcomes. Hence, any difference in speed-density relationship at upstream and downstream must be attributed only to the presence of bottleneck.

\section{Site Description and Data Collection}

The video graphic data was collected at the Mahakaleshwar Temple, which is the main attraction in the city of Ujjain, India. The data was collected at the temple on Mahashivaratri, a festival day on which large number of pilgrims visited the temple. Makeshift corridors were temporarily installed at the entrance of temple to channelize the crowd. Pedestrians were made to walk in long, narrow corridors in order to accommodate more pedestrians and thereby increase the capacity. A GoPro camera was installed right above the pedestrian flow so as to capture two adjacent corridors, where width of the corridor reduces from $1.6 \mathrm{~m}$ to $0.75 \mathrm{~m}$ at the U-turn. Figure 1 shows the study area enclosed by a rectangle and the direction of pedestrian flow. Figure 2 shows the screenshot of both the study sections. The reduction in width has led to the formation of bottleneck at the U-turn. The video had captured both upstream and downstream of the bottleneck simultaneously. The length of both the study sections below the camera are $2.3 \mathrm{~m}$. The corridors are separated by strong barricades. Pedestrians can see the crowd in front as well as in the adjacent corridors through the barricades to get visual clues of pedestrian flow on the other side i.e. pedestrians at upstream can see pedestrian flow at downstream. A video of 38 minutes was analyzed in which nearly 2300 pedestrians (flow rate $3600 \mathrm{ped} / \mathrm{hr}$ ) passed through the study sections.

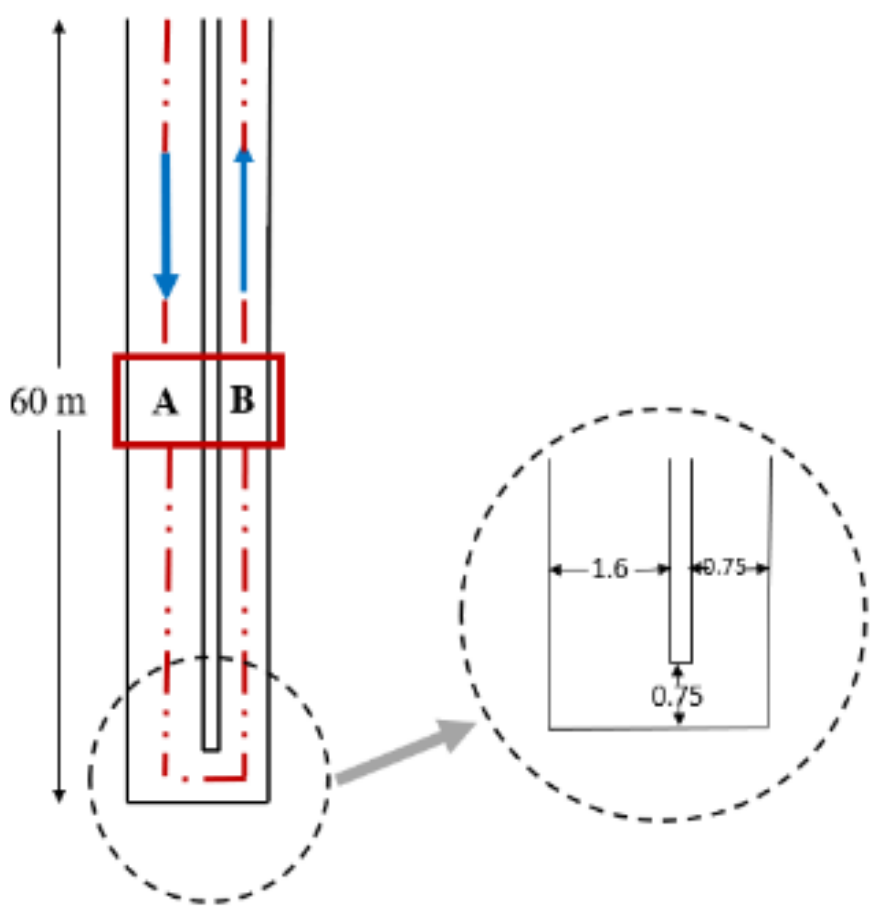

Figure 1 Schematic diagram of pedestrian flow (all dimensions are in meters)

Traps of known dimensions were annotated on the video using Swordsoft Screenink tool to mark the study area and entry and exit times of all the pedestrians passing through the study sections were extracted manually. The entry and exit time of all pedestrians were recorded from video using head as the reference 
i.e. a pedestrian was considered to have entered the study section, if at least half of the head of a pedestrian is inside. Similarly, the exit times were also recorded. The entry and exit times were used to calculate the speed of individual (which later gave average speed) and density at every second. Therefore, density has a limitation that it can only take discrete values, based on the number of pedestrians in the study area. The modified density, as mentioned in equation 1 of [13] was chosen, which is as follows.

$$
\Theta_{i}(t)=\left\{\begin{array}{cl}
\frac{t-t_{i}^{i n}}{t_{i+1}^{\text {in }}-t_{i}^{\text {in }}} & : t \in\left[t_{i}^{\text {in }}, t_{i+1}^{\text {in }}\right] \\
1 & : t \in\left[t_{i+1}^{\text {in }}, t_{i}^{\text {out }}\right] \\
\frac{t_{i+1}^{\text {out }}-t}{t_{i+1}^{\text {out }}-t_{i}^{\text {out }}} & : t \in\left[t_{i}^{\text {out }}, t_{i+1}^{\text {out }}\right] \\
0 & : \text { otherwise }
\end{array}\right.
$$

Where,

$$
\begin{array}{ll}
\Theta_{i}(t) & =\text { fraction to which the space between person } i \text { and person } i+1 \text { is inside } \\
t_{i}^{\text {in }} & =\text { entrance time } \\
t_{\text {in }}^{\text {out }} & =\text { exit time }
\end{array}
$$

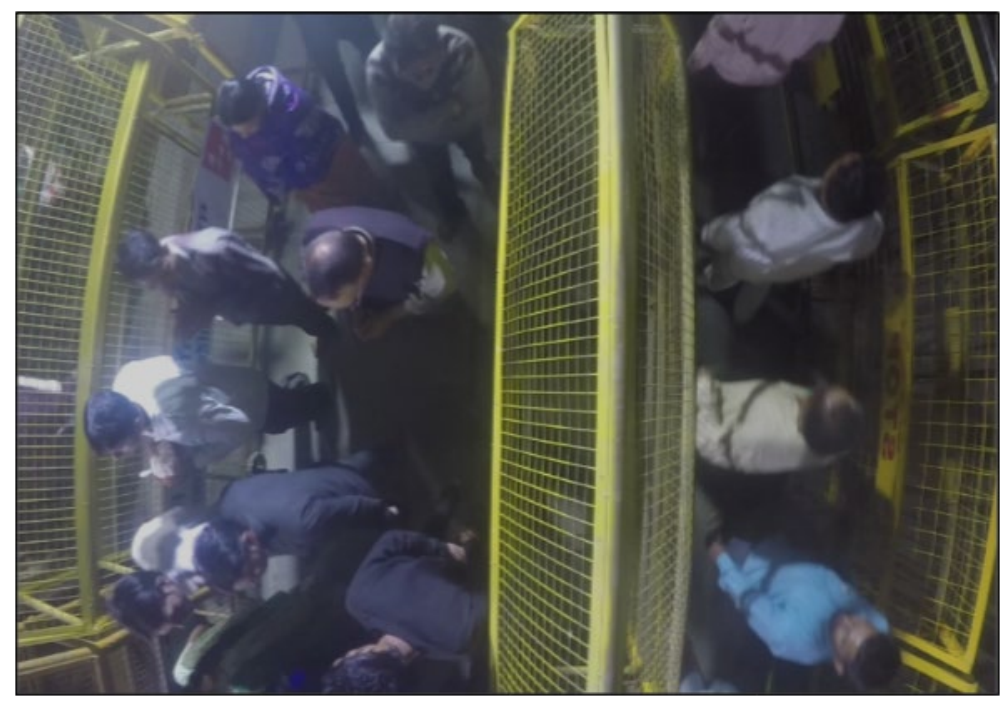

Figure 2 Screenshot of the study area from overhead video

\section{Data Analysis}

The speed-density relationship can be used to quantitatively analyze any pedestrian facility. The inter-quartile graph of speed-density relationship for upstream and downstream section were plotted as shown in Figure 3. (The inter-quartile graphs did not change its behavior for varied range of bin size and bin positions).

In speed-density relationship of upstream, free flow speeds are achieved at low density, which is logical and as expected. For density more than $1 \mathrm{ped} / \mathrm{m}^{2}$, the speed of the pedestrians showed a sudden drop followed by a gradual decrease. The lack of intermediate speed points in the graph can also be visualized with help of Figure 4. For density more than $1 \mathrm{ped} / \mathrm{m}^{2}$, speed values are very low, even for the intermediate density values. The region of low speed stretches from intermediate to high values of density. This suggests that pedestrians in the upstream are either at free flow speed for very low density values or moving slowly for intermediate to high range of density values. The period of transition between free flow to congestion is sudden. From the speed-density relationship, it can be concluded 
that pedestrians at upstream had visual clues of congestion ahead at bottleneck (pedestrian could also see the downstream flow through barricades). Therefore, pedestrians wait at their position, stay in their comfort zone and do not push each other. Thus, even at intermediate local density, pedestrians have such low speeds.

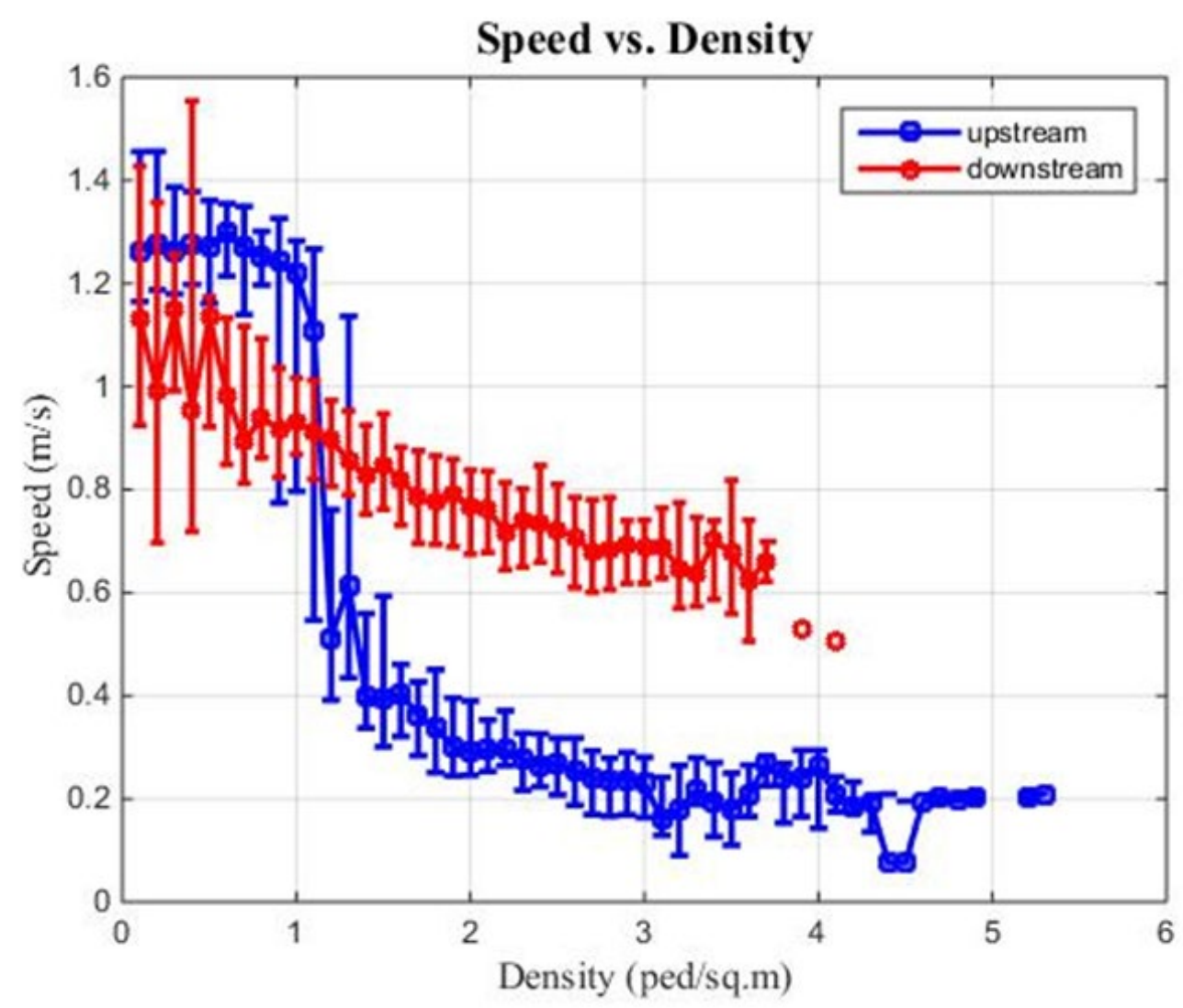

Figure 3 Speed-Density relationship for upstream and downstream section

After crossing bottleneck, pedestrians enter into the downstream section. There were no bottlenecks or obstacles ahead. The free flow speed values are slightly lower because of the presence of side friction from barricades. Since, the channel was very narrow; pedestrians could only enter one after the other from bottleneck section. In downstream, pedestrian had to walk in single file due to constrained motion in the lateral direction. However, the rate of decrease of speed with increase in density was very less. One of the reason is pedestrians had to compensate for the delay at bottleneck. Hence, even increased density could not reduce the speed as expected or mentioned in literature. The density mainly varied between $1 \mathrm{ped} / \mathrm{m}^{2}$ to $2 \mathrm{ped} / \mathrm{m}^{2}$, the histogram of density can be seen in Figure 4 . The high peaks between these ranges, all indicates that bottleneck was saturated for most of the time. 

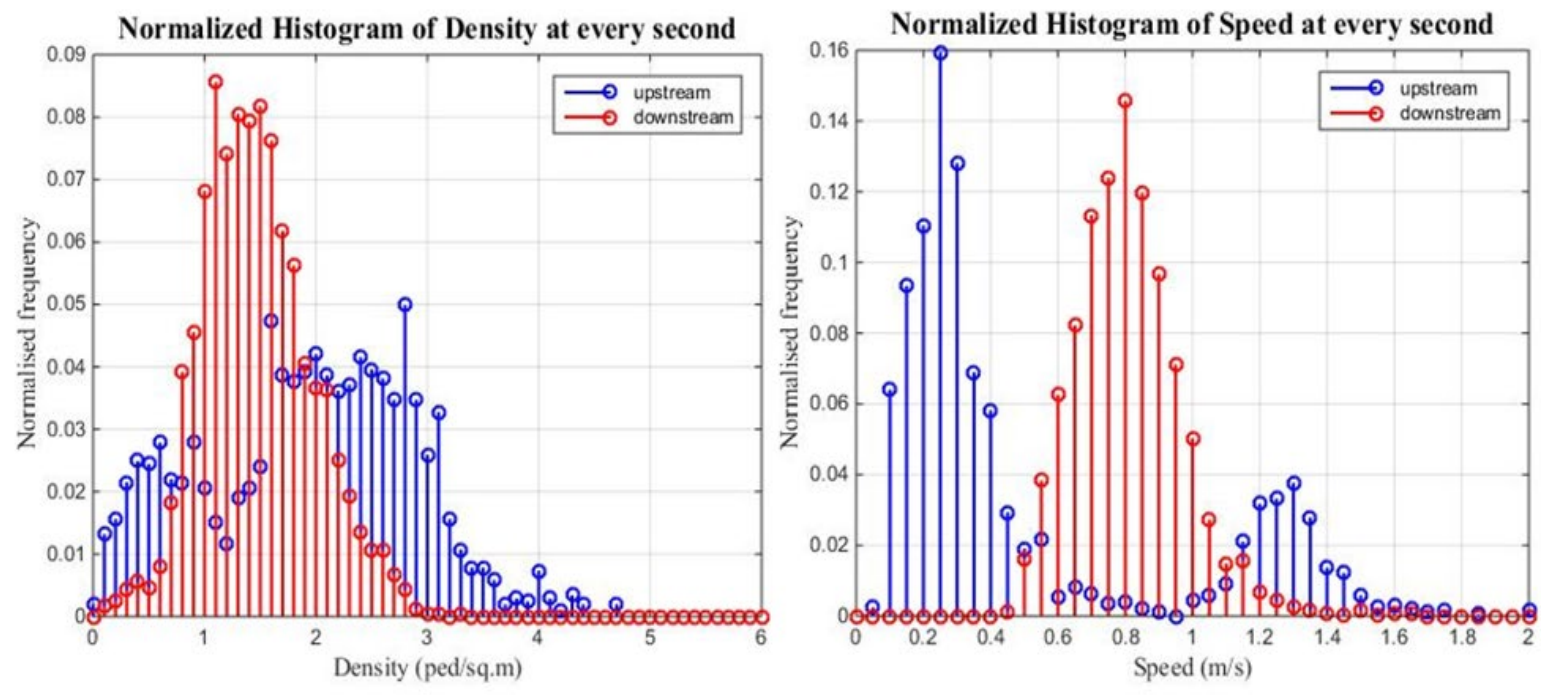

Figure 4 Frequency of density and speed values as upstream and downstream

\section{Conclusion}

The paper observes the continuous real pedestrian flow characteristics at upstream and downstream of the bottleneck. It quantitatively shows that pedestrian flow characteristics at upstream is different from that of downstream. It can be concluded that pedestrian flow in downstream is governed not only by local factors like density and side friction, but also by pedestrian's willingness to compensate for the delay occurred due to bottleneck. On the other hand, slow moving speed at intermediate density suggest that pedestrian flow at upstream is not only governed by density but possibly also by other factors such as visual clues of congestion status ahead. The results are limited to the pedestrian flow at normal condition; there was no state of panic in the crowd. The study also hints that a single speed-density relationship does not apply to the entire length of the section. There are factors, apart from density, which affects the flow characteristics. The study also suggest that pedestrians in upstream anticipate the visible changes in downstream and slow down. The assumption that speed of pedestrians will reduce only at shockwave interface is also violated. The paper compares the upstream and downstream section characteristics of continuous pedestrian flow, at a macroscopic level, which may have certain limitations. The results can be used for calibration and validation of simulations involving pedestrian movement through long and narrow makeshift corridors for crowd gathering events. In future, comparison based on microscopic characteristics can yield more contrasting differences between upstream and downstream characteristics.

\section{Acknowledgements}

The work reported in this paper is part of the project titled "The Kumbh Mela Experiment: Measuring and Understanding the Dynamics of Mankind's largest crowd," funded by the Ministry of Electronics and IT Ministry of Communication and Information Technology, Government of India (MITO-0105), Netherlands Organization for Scientific Research, NWO (Project no. 629.002.202), and Robert Bosch Center for Cyber Physical Systems, Indian Institute of Science, Bangalore. (Grant No. RBCO001). The authors also express their gratitude towards Kumbh Mela administration and government of Madhya Pradesh, India for providing constant support and official permissions to carry out research work and establish Indo-Dutch collaboration research camp at Kumbh Mela 2016. 


\section{References}

[1] Kretz, T., Grunebohm, A., \& Schreckenberg, M. (2006, October). Experimental study of pedestrian flow through a bottleneck. Jounal of Statistical Mechanics: Theory and Experiment.

[2] Daamen, W., \& Hoogendoorn, S. P. (2003). Experimental Research of Pedestrian Walking Behavior. Transportation Research Record, 1828, 20-30.

[3] Rupprecht, T., Klingsch, W., \& Seyfried, A. (2011). Influence of Geometry Parameters on Pedestrian Flow through Bottlenecks. Pedestrian and Evacuation Dynamics (pp. 71-80). Springer.

[4] Daamen, W., \& Hoogendoorn, S. (2010). Emergency Door Capacity: Influence of Population Composition and Stress Level. Fire Technology, 48(1), 55-71.

[5] Duives, D., Daamen, W., \& Hoogendoorn, S. (2014). Anticipation behavior upstream of a bottleneck. Transportation Research Procedia, 2, pp. 43-50.

[6] Virkler, M. R., \& Elayadath, S. (1994). Pedestrian Density Characteristics and Shockwaves. Proceedings of the Second International Symposium on Highway Capacity, (pp. 671-684).

[7] Tarawneh, M. S. (2001). Evaluation of pedestrian speed in Jordan with investigation of some contributing factors. Journal of Safety Research, 32, 229-236.

[8] Rastogi, R., Ilango, T., \& Chandra, S. (2013). Pedestrian flow characteristics for different pedestrian facilities and situations. European Transport, 53, 5.

[9] Chattaraj, U., Seyfried, A., \& Chakroborty, P. (2009). Comparison of pedestrian fundamental diagram across cultures. Advance in Complex Systems, 12, 393.

[10] Older, S. J. (1968). The speed, density and flow of pedestrians on footway in shopping streets. Traffic Engineering and Control, 10(4), 160-163.

[11] Lam, H. K., Morall, J. F., \& Ho, H. (1995). Pedestrian flow characteristics in Hong Kong. Transportation Research Record, 1487, 56-62.

[12] Ye, J., Chen, X., \& Jian, N. (2012). Impact analysis of human factors on pedestrian traffic characteristics. Fire Safety Journal, 52, 46-54.

[13] Seyfried, A., Steffen, B., Klingsch, W., \& Boltes, M. (2005). The fundamental diagram of pedestrian movement revisited. Journal of Statistical Mechanics, P10002. 